J. Lake Sci. (湖泊科学), 2012, 24(3): 416-421

http: //www.jlakes.org. E-mail : jlakes@niglas.ac.cn

(C) 2012 by Journal of Lake Sciences

\title{
湿地植物种类和生长方式对根际酞酸酯及其单酯代谢物分布特征的 影响
}

迟 杰, 杨 瑞, 王爱丽

(天津大学环境科学与工程学院,天津 300072)

摘 要: 选取天津大学校内两个相邻的小型湖泊 (青年湖和爱晚湖) 为研究对象, 研究了湿地植物种类 (芦苇和香蒲) 和 生长方式 (单生和混生) 对根际唒酸酯 (PAEs) 及其单酯代谢物 (PAMs) 分布特征的影响. 结果显示: 几乎所有的样品中都 能检测到唒酸丁二酯 (DBP) 和唒酸二异辛酯 (DEHP) 及其单酯代谢物 (MBP 和 MEHP), 并且 DBP 和 DEHP 的浓度比相 应的单酯代谢物高 $1 \sim 2$ 个数量级; 沉积物和根样品中 DEHP 的浓度高于 DBP; 芦苇和香蒲根对 DEHP 的富集系数 $(R C F)$ 大于 DBP. 当芦苇和香蒲单生时 (即青年湖), 芦苇根际沉积物中 DBP 和 DEHP 浓度 $\leqslant$ 香蒲根际沉积物的浓度, 并且芦苇 根的 $R C F$ 值也小于香蒲. 当芦苇和香蒲混生时 (即爱晚湖), 芦苇根际沉积物中 DBP 和 DEHP 浓度 <香蒲根际沉积物的 浓度, 而芦苇根的 $R C F$ 值 $\geqslant$ 香蒲根的 $R C F$ 值, 与青年湖中不同. 青年湖香蒲根际沉积物中 DBP 和 DEHP 的浓度小于爱晚 湖, 而香蒲根的 $R C F$ 值与此刚好相反. 芦苇根对这两种 PAEs 的富集没有这种关系, 可能是由于这两个湖中芦苇的生长状 况不同, 与香蒲混生时, 芦苇的生长受到抑制. 对 PAEs 的单酯代谢产物分析发现, 沉积物中 MBP/DBP > MEHP/DEHP. MEHP 在根中的浓度多数情况高于 MBP, 表明 MEHP 比 MBP 具有较强的根富集能力. 同种植物单生时, 根中 PAM 浓度均 $\geqslant$ 混生.

关键词: 唒酸酯;单酯代谢物;芦苇;香蒲;根际

\section{Effect of wetland plant species and growth strategy on the distribution of PAEs and their monoester metabolites in the rhizosphere}

\author{
CHI Jie, YANG Rui \& WANG Aili \\ (School of Environmental Science and Engineering, Tianjin University, Tianjin 300072, P. R. China)
}

\begin{abstract}
The field study was conducted in two adjacent shallow ponds (Lake Qingnian and Lake Aiwan) located within the campus of Tianjin University. The effect of wetland plant species (Phragmites australis and Typha orientalis) and growth strategy ( single or mix) on the distribution of phthalic acid esters (PAEs) and their monoester metabolites (MBP and MEHP) were investigated. The results showed that dibutyl phthalate (DBP), ethylhexyl phthalate (DEHP) and their monoester metabolites were detected in nearly all of the samples and concentrations of DBP and DEHP were $1-2$ order of magnitude higher than those of their corresponding monoester metabolites. The facts that concentrations of DEHP in sediments and root were higher than that of DBP, and root concentration factor $(R C F)$ of DEHP was higher than that of DBP suggest that when P. australis and T. orientalis grew alone (i. e. Lake Qingnian), concentrations of DBP and DEHP in rhizosphere sediments were no more than those in non-rhizosphere sediments; concentrations of DBP and DEHP in the rhizosphere of $P$. australis were no more than those in the rhizosphere of T. orientalis, and RCF values of the two PAEs by $P$. australis were also lower than those by $T$. orientalis. When $P$. australis and T. orientalis grew together (i. e. Lake Aiwan), concentrations of DBP and DEHP in the rhizosphere of $P$. australis were lower than those in the rhizosphere of $T$. orientalis, while $R C F$ values of the two PAEs by $P$. australis were higher than those by $T$. orientalis. This is different from that in Lake Qingnian. Concentrations of DBP and DEHP in the rhizosphere of $P$. australis from Lake Qingnian were lower than those from Lake Aiwan, while T. orientalis RCF values in Lake Qingnian were higher than those in Lake Aiwan. However, this phenomenon was not observed in the PAE accumulation by $P$. australis, which may be due to the different
\end{abstract}

* 国家自然科学基金项目(20777052)资助. 2011-07-27 收稿;2011-10-24 收修改稿. 迟杰,女,1964 年生,博士, 教授;E-mail:cjiechi@yahoo.com. 
growth conditions of $P$. australis in the two lakes during the sampling period. The growth of $P$. australis was significantly inhibited when the two plants grew together. Results of monoester metabolites of the two PAEs showed that concentration ratio of MBP to DBP was higher than that of MEHP to DEHP. Concentrations of MEHP were mostly higher than those of MBP, showing stronger root accumulation of MEHP than MBP. PAM concentrations in roots under the condition of growing separately were higher than those under the condition of growing together.

Keywords: PAEs; monoester metabolites; Phragmites australis; Typha orientalis; rhizosphere

酞酸酯 ( 又名邻苯二甲酸酯, PAEs), 是邻苯二甲酸酤与醇经酯化反应生成的化合物, 被广泛应用于塑料 的增塑剂和软化剂, 其中使用最多的是酞酸二异辛酯 (DEHP), 其次是酞酸丁二酯 (DBP). 由于塑料制品在 工农业生产和日常生活中广泛使用, 产量不断增加. 目前 PAEs 已成为全球最普遍存在的环境污染物. 酞酸 单酯 (PAMs) 是酞酸酯的初级代谢产物. 近些年的研究发现, 酞酸单酯可产生多种扰乱动物内分泌的生化和 整体效应并对生殖系统产生损伤, 毒性明显高于其母体化合物 ${ }^{[1]}$.

在水体环境中, 沉积物通常是 PAEs 等疏水性有机物 (HOCs) 的汇. 由于疏水性,HOCs 含量主要与沉积物中的 有机质有关 ${ }^{[2]}$. 湿地植物具有发达的根系, 可利用其根际修复受 HOCs 污染的沉积物. 研究表明湿地植物如芦苇、 水葱等的根可以富集沉积物中的有机氯农药 ${ }^{[3-4]}$. 植物根际还能明显促进有机污染物的微生物降解 ${ }^{[5-6]}$. 芦苇和香 蒲是两种典型的湿地植物, 遍布于世界各地, 生长在灌溉沟渠旁、河堤沼泽地等, 是人工湿地和河岸缓冲带常用的 植物. 在自然生态系统中, 芦苇和香蒲通常单生或混生. 近年来, 常采用混合植物群落修复受污染的水体 ${ }^{[7]}$. 本文以 芦苇和香蒲为研究对象, 以天津大学两个相邻的浅水湖为现场, 采样调查了植物种类和生长方式对植物根际 PAEs 及其单酯代谢物分布的影响, 为沉积物中这类污染物的植物修复提供数据支持.

\section{1 现场描述和样品采集}

本研究采样点位于天津大学校内的两个相邻的小型湖泊 (青年湖和爱晚湖), 两个湖中均生长着大量的 挺水植物, 包括芦苇和香蒲. 青年湖中香蒲和芦苇各自形成单一优势群落 (即单生) ; 爱晚湖中香蒲和芦苇交 错生长 (即混生), 其中香蒲生长情况与青年湖相似, 而芦苇生长受到明显抑制, 平均株高约为青年湖芦苇的 1/2. 于 2010 年 5 月 18 日采集两个湖中芦苇和香蒲的根、根际和非根际沉积物. 根际沉积物采用抖根法采 集, 具体做法是将根上大量的沉积物抖掉, 附着在根表面且不脱落的沉积物作为根际沉积物. 青年湖中设 3 个采样区 (芦苇区、香蒲区、无植物区), 爱晚湖中选取 2 个采样区 (芦苇-香蒲混生区和无植物区). 在每个采 样区内每种样品 (即香蒲和芦苇根及根际沉积物、非根际沉积物) 采用五点取样法, 按梅花形取 5 个样方 (50 $\mathrm{cm} \times 50 \mathrm{~cm}$ ). 采集的样品 (根及沉积物) 装在干净密封的聚四氟乙烯塑料袋中, 于 $0 \sim 4^{\circ} \mathrm{C}$ 下保存并及时送回 实验室, 等量混合后最终成为一个样品. 沉积物样品真空冷冻干燥后, 用于 PAEs 及 PAMs 分析, 植物样品于 $4{ }^{\circ} \mathrm{C}$ 冰箱保存, 尽快测定.

\section{2 样品准备和分析}

\section{1 PAEs 及 PAMs 分析}

将采来的植物根先用自来水冲洗, 再用蒸馏水洗, 吸干表面水分后, 称取 $1 \mathrm{~g}$ (湿重), 剪碎, 放人组织研 磨器, 加人 $1 \mathrm{ml} 2 \mathrm{~mol} / \mathrm{L} \mathrm{HCl}$, 加人 $5 \mathrm{ml} \mathrm{CH} \mathrm{Cl}_{2}$, 研磨 $5 \mathrm{~min}$, 移出植物匀浆至离心管中 (移取时先用少量 $\mathrm{CH}_{2} \mathrm{Cl}_{2}$ 淋洗, 再用适量水淋洗). $4500 \mathrm{r} / \mathrm{min}$ 离心 $5 \mathrm{~min}$, 先去掉上层水相, 然后用胶头滴管吸取 $\mathrm{CH}_{2} \mathrm{Cl}_{2}$ 层到 K-D 浓缩器中, 在 $\mathrm{N}_{2}$ 吹脱下浓缩至干燥. 硅烷化试剂衍生后测定 PAEs 及 PAMs, 每次 3 个平行. 衍生化参考 Jonsonn 的方法 ${ }^{[8]}$.

取 $1 \mathrm{~g}$ 沉积物 (冷冻干燥后研磨过 80 目笁) 于 $10 \mathrm{ml}$ 离心管中, 加人 $3 \mathrm{ml} \mathrm{CH} \mathrm{Cl}_{2}$, 超声萃取 $15 \mathrm{~min}$, 然后 $4000 \mathrm{r} / \mathrm{min}$ 离心 $5 \mathrm{~min}$, 将上清液倒人 $10 \mathrm{ml} \mathrm{K}-\mathrm{D}$ 浓缩管中, 重复 3 次, 合并有机相. 在 $\mathrm{N}_{2}$ 吹脱下浓缩至 $1 \mathrm{ml}$. 提取完 PAEs 的沉积物用来继续提取 PAMs: 向沉积物中加人 $3 \mathrm{ml} 0.01 \mathrm{~mol} / \mathrm{L} \mathrm{HCl}$, 超声萃取 $15 \mathrm{~min}$, 然后 $4000 \mathrm{r} / \mathrm{min}$ 离心 $5 \mathrm{~min}$, 倾出上清液于 $50 \mathrm{ml}$ 比色管中, 用 $2 \mathrm{~mol} / \mathrm{L} \mathrm{HCl}$ 调 $\mathrm{pH}$ 至 2 , 加人 $3 \mathrm{ml} \mathrm{CH}_{2} \mathrm{Cl}_{2}$, 振摇 $10 \mathrm{~min}$, 静置 $5 \mathrm{~min}$. 用胶头滴管将 $\mathrm{CH}_{2} \mathrm{Cl}_{2}$ 层移人 $10 \mathrm{ml} \mathrm{K}-\mathrm{D}$ 浓缩管, 在 $\mathrm{N}_{2}$ 吹脱下浓缩至干燥, 用硅烷试剂衍 生. 每次做 3 个重复. 
处理后的样品用 Agilent 6890N/5975C 气质联用仪测定 (配石英毛细管柱 HP-5MS). 气相炉温的升温程 序为: 初始温度 $130^{\circ} \mathrm{C}$, 保持 $1 \mathrm{~min}$, 以 $25^{\circ} \mathrm{C} / \mathrm{min}$ 升到 $280^{\circ} \mathrm{C}$, 保持 $5 \mathrm{~min}$. 载气 $\mathrm{He}$ 流量为 $1.0 \mathrm{ml} / \mathrm{min}$, 不分流 进样 $1 \mu \mathrm{l}$, 进样口和检测器温度均为 $250^{\circ} \mathrm{C}$, 接口温度 $280^{\circ} \mathrm{C}, \mathrm{EI}$ 源 $70 \mathrm{eV}$. GC-MS 选用 SIM 模式分析, 内标法 进行定量 (以间苯二甲酸二甲酯作为内标). PAEs 和 PAMs 硅烷化衍生物的停留时间和所选择的质谱片段 见表 1. 根中浓度以湿重计, 沉积物中浓度以干重计.

定期对仪器设备的稳定性进行检查, 确定目标污染物质的色谱保留时间及其灵敏度满足分析方法的要 求. 所有的数据都经过了严格的质量控制程序, 对于一系列的样品分析, 均运行溶剂空白和方法空白, 以检 查干扰和污染. 沉积物中 DBP 和 DEHP 的加标回收率分别为 $98.3 \%$ 和 $95.0 \%$, 相对标准偏差 $(R S D)<$ $15.0 \%$; 根中 DBP 和 DEHP 的加标回收率分别为 $94.1 \%$ 和 $92.8 \%, R S D<9.6 \%$. 沉积物中 DBP 和 DEHP 的 单质代谢物酞酸单丁酯 (MBP) 和酞酸单异辛酯 (MEHP) 的加标回收率分别为 $92.5 \%$ 和 $95.0 \%, R S D<$ $11.7 \%$; 根中 MBP 和 MEHP 的加标回收率分别为 $95.2 \%$ 和 $104.1 \%, R S D<5.7 \%$.

表 1 PAEs 和 PAMs 硅烷化衍生物的停留时间和选择性离子扫描的质谱碎片

Tab. 1 Retention time and mass selective ion scan of debris of PAEs and silylated derivatives of PAMs

\begin{tabular}{ccccc}
\hline 化合物 & 英文缩写 & 停留时间 $/ \mathrm{min}$ & 分子量/D & 离子碎片 $/(\mathrm{m} / \mathrm{z})$ \\
\hline 酞酸间二甲酯 & $\mathrm{DMiP}$ & 5.36 & 194 & $163^{\mathrm{a}}, 149,194$ \\
酞酸单丁酯 & MBP & 6.95 & 294 & $223^{\mathrm{a}}, 221,149,279$ \\
酞酸二丁酯 & DBP & 7.64 & 278 & $149^{\mathrm{a}}, 205,223$ \\
酞酸单异辛酯 & MEHP & 8.35 & 350 & $221^{\mathrm{a}}, 223,149,239$ \\
酞酸二异辛酯 & DEHP & 11.20 & 390 & $149^{\mathrm{a}}, 167,279$ \\
\hline
\end{tabular}

a代表定量离子.

\section{2 其他指标的测定}

水和沉积物样品的物理化学特性测定包括: 水的 $\mathrm{pH}$ 、总氮 ( TN) 和总磷 (TP), 沉积物的 $\mathrm{pH}$ 、总有机碳 (TOC) 含量 ${ }^{[9]}$. 根样品测定脂肪含量, 采用石油醚提取 ${ }^{[10]}$.

\section{3 数据分析}

应用 SPSS 13.0 软件对实验数据进行单因素方差分析 (ANOVA), 即在平均值比较基础上采用 LSD (Least Significant Difference) 方法,在 $a=0.05$ 水平进行数据差异显著性检验.

常用根富集系数 (Root Concentration Factor, $R C F$ ) 来反映植物根对污染物的富集能力. $R C F$ 为根中的污 染物浓度除以根际沉积物中污染物浓度.

\section{3 结果}

\section{1 水体和沉积物的物理化学性质}

青年湖水中 TN、TP 浓度大约是爱晚湖的 2 倍, 营养盐的污染相对较重. 这使得青年湖非根际沉积物中 TOC 含量略高于爱晚湖非根际沉积物; 两个湖中根际沉积物的 TOC 含量均高于非根际. 两个湖水 $\mathrm{pH}$ 值相 差不大;根际沉积物 $\mathrm{pH}$ 值略小于非根际 (表 2).

表 2 水和沉积物的物理化学特性、根的脂肪含量

Tab. 2 Physical and chemical properties of water and sediment, and lipid content of root

\begin{tabular}{|c|c|c|c|c|c|c|c|}
\hline \multirow{2}{*}{ 湖泊 } & & \multicolumn{3}{|c|}{ 水 } & \multicolumn{2}{|c|}{ 沉积物 } & \multirow{2}{*}{$\begin{array}{c}\text { 根的脂肪含量/\% } \\
\text { (以湿重计) }\end{array}$} \\
\hline & & $\mathrm{pH}$ & $\mathrm{TN} /(\mathrm{mg} / \mathrm{L})$ & $\mathrm{TP} /(\mathrm{mg} / \mathrm{L})$ & $\mathrm{pH}$ & TOC/\% & \\
\hline \multirow[t]{3}{*}{ 青年湖 } & 香蒲根际 & 8.9 & 9.28 & 0.040 & 7.0 & 2.40 & 0.50 \\
\hline & 芦苇根际 & & & & 7.3 & 3.49 & 0.43 \\
\hline & 非根际 & & & & 7.6 & 2.20 & \\
\hline \multirow[t]{3}{*}{ 爱晚湖 } & 香蒲根际 & 8.8 & 5.85 & 0.020 & 7.2 & 8.17 & 0.24 \\
\hline & 芦苇根际 & & & & 7.4 & 2.54 & 0.37 \\
\hline & 非根际 & & & & 7.5 & 1.53 & \\
\hline
\end{tabular}




\section{2 沉积物及植物根中 PAEs 浓度}

所有样品中均检出 DBP 和 DEHP. 沉积物中 DBP、DEHP 的浓度分别为 $0.066 \sim 0.180 \mu \mathrm{g} / \mathrm{g}$ 和 $0.32 \sim$ $0.76 \mu \mathrm{g} / \mathrm{g}$. 两个湖中香蒲根际沉积物中 DBP 和 DEHP 的浓度均高于芦苇根际. 两个湖中 DBP 和 DEHP 具有 不同的分布特征, 青年湖中芦苇和香蒲单生, DBP 与 DEHP 分布特征不同, DBP 为非根际沉积物 > 香蒲根际 沉积物 $\approx$ 芦苇根际沉积物, DEHP 为非根际沉积物 $\approx$ 香蒲根际沉积物 $>$ 芦苇根际沉积物. 芦苇和香蒲混生 时, DBP 和 DEHP 的分布特征相同, 为香蒲根际沉积物 > 芦苇根际沉积物 $\approx$ 非根际沉积物 (图 1).

根样品间的脂肪含量 (以湿重计) 差异不大 (大约在 2 倍以内) ; 单生时香蒲和芦苇根的脂肪含量略高于 混生时根的脂肪含量. 香蒲、芦苇根中 DBP 和 DEHP 的浓度 (以湿重计) 表明, 所有样品中均检出 DBP 和 DEHP, 浓度分别为 $0.034 \sim 0.054 \mu \mathrm{g} / \mathrm{g}$ 和 $0.24 \sim 0.61 \mu \mathrm{g} / \mathrm{g}$ (图 2).

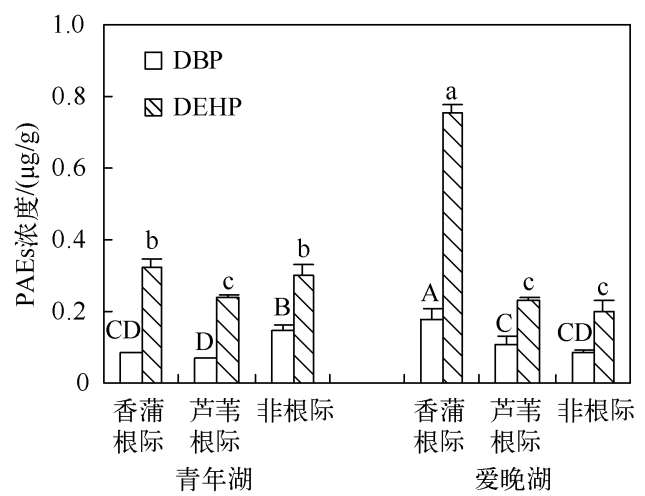

图 1 沉积物中 PAEs 浓度

(具有相同字母表示差异不显著, $P>0.05$,下同)

Fig. 1 PAEs concentrations in sediment

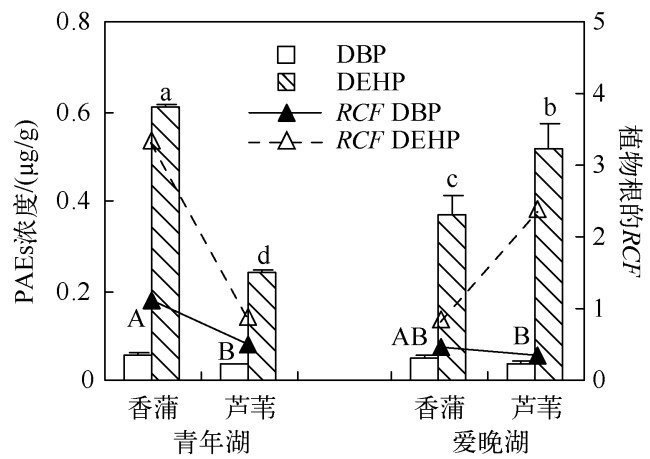

图 2 植物根中 PAEs 浓度及 $R C F$

Fig. 2 PAEs concentrations and $R C F$ in roots

\section{3 沉积物及植物根中 PAMs 浓度}

除爱晚湖非根际沉积物外, 其余样品中 MBP 和 MEHP 均有检出, 沉积物中 MBP、MEHP 的浓度分别为 $0 \sim$ $11.99 \mathrm{ng} / \mathrm{g}$ 和 $0 \sim 23.75 \mathrm{ng} / \mathrm{g}$, 比相应的双酯低 $1 \sim 2$ 个数量级; 芦苇和香蒲根所有样品中均检出 MBP 和 MEHP, 浓度分别为 $1.87 \sim 6.05 \mathrm{ng} / \mathrm{g}$ 和 $3.7 \sim 14.7 \mathrm{ng} / \mathrm{g}$, 比相应 PAEs 低 1 2 个数量级 (图 3 ).
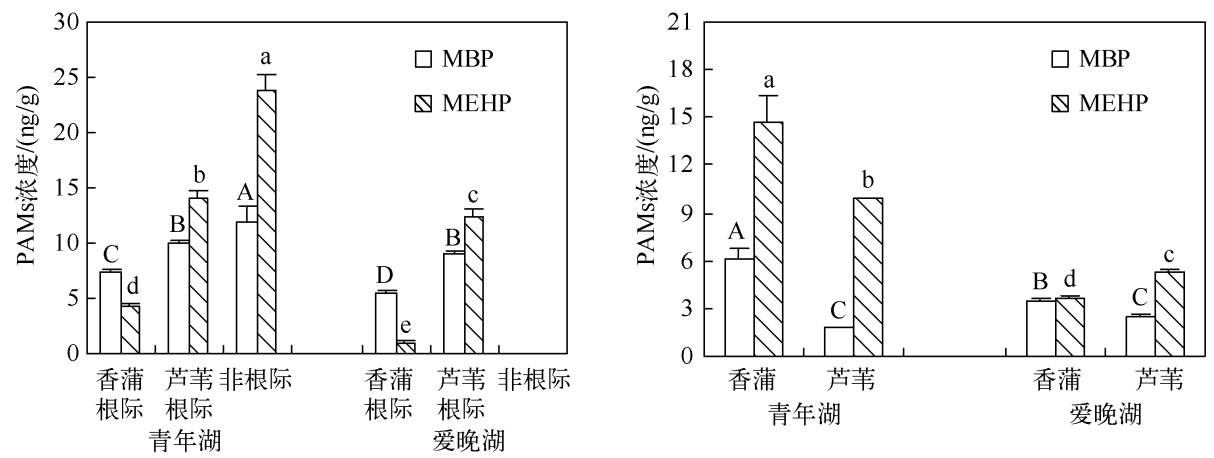

图 3 沉积物和植物根中 PAMs 浓度

Fig. 3 PAMs concentrations in sediment and roots

\section{4 讨论}

\section{1 PAEs 的分布特征}

青年湖和爱晚湖的根际和非根际沉积物样品中, DEHP 的浓度均高于 DBP, 这主要是因为 DEHP 的辛 醇-水分配系数 $\left(K_{\mathrm{OW}}\right)$ 较高, 疏水性较强, 使 DEHP 更容易吸附在沉积物上, 降低了其生物有效性. 同样, 
DEHP比 DBP 易富集在根中, 浓度高于 DBP. 经计算, 根对 DEHP 的 $R C F$ 值显著大于 DBP(图 2).

本研究表明, 根际沉积物的 TOC 含量大于非根际沉积物 (表 2), 与文献报道一致 ${ }^{[11-12]}$. 根在长期生长过 程中不断向根际分泌有机物并脱落根毛和根表皮细胞, 是根际沉积物有机质含量较高的原因. 另外, 根际微 生物量较大可能也是原因之一. 已有的研究表明, 沉积物中 HOCs 的分布主要受沉积物中 TOC 含量的限制, 有机质含量高的沉积物吸附或固定 HOCs 的量大 ${ }^{[13-14]}$. Srivastava 等也发现 PAEs 的浓度分布随沉积物中 TOC 含量的增加而增加 ${ }^{[15]}$. 因此, 根际沉积物中较高的 TOC 含量容易吸附较高浓度的 PAEs, 然而现场采样 数据并非如此.

在青年湖中,芦苇和香蒲各自单生. 根际沉积物中 PAEs 浓度均小于或等于非根际沉积物, 尤其是 DBP, 明显小于非根际沉积物中的浓度, 说明芦苇和香蒲单生时均能够有效去除根际沉积物中的 PAEs. 植物去除 根际沉积物中污染物的机制主要包括根际微生物对污染物的降解及根对污染物的吸附和吸收. 比较芦苇和 香蒲根际 PAEs 浓度可以看出, 虽然芦苇根际 TOC 含量高于香蒲, 但是芦苇根际 PAEs 含量反而低于香蒲. 香蒲根对 DBP 和 DEHP 的富集能力大于芦苇根, $R C F$ 值分别是芦苇根的 1.1 和 1.9 倍. 这说明芦苇根际较 低的 PAE 浓度可能是由较高的根际微生物活性所致.

在爱晚湖中, 芦苇和香蒲混生. 香蒲根际沉积物中 DBP 和 DEHP 的浓度高于非根际沉积物, 分别是非根 际沉积物的 2.0 和 3.8 倍; 香蒲根际沉积物 TOC 含量也明显高于非根际沉积物, 是非根际沉积物的 5.3 倍. 芦苇根际沉积物中 PAEs 浓度与非根际没有显著性差异, 但是芦苇根际沉积物的 TOC 含量略高于非根际沉 积物, 是非根际沉积物的 1.6 倍. 这说明芦苇和香蒲混生时对根际沉积物中 PAEs 也具有一定的去除作用, 尤其是对香蒲根际 DBP 的作用最为显著. 芦苇根对 DBP 的 RCF 值与香蒲相近, 对 DEHP 的 RCF 值是香蒲 的 4.6 倍. 由此可以推测, 香蒲根际微生物活性较芦苇根际高, 与青年湖中正好相反. 这可能是芦苇与香蒲 混生时芦苇的生长受到抑制, 根际效应较弱的原因.

青年湖香蒲根际沉积物中 DBP 和 DEHP 的浓度均小于爱晚湖香蒲,而青年湖香蒲根对这两种 PAEs 的 $R C F$ 值均大于爱晚湖香蒲, 即根际沉积物中 PAEs 浓度较高时, 植物根的 RCF 值反而偏低, 这与 Liu 等研究 水葱对 HCH 和 DDT 的植物富集时的结果一致 ${ }^{[3]}$. 芦苇根对这两种 PAEs 的富集没有这种关系, 这可能是由 于这两个湖中芦苇的生长状况不同,与香蒲混生时,芦苇的生长受到抑制.

\subsection{PAMs 的分布特征}

PAEs 的生物降解途径是首先水解生成相应的 PAMs, PAMs 可进一步被酶水解生成邻苯二甲酸, 邻苯二 甲酸可进一步被降解成苯甲酸, 最终生成二氧化碳和水 ${ }^{[2]}$. 因此, 环境中 PAMs 的浓度分布更复杂. 本研究大 部分沉积物中 MEHP 的浓度高于 MBP. Otton 等 ${ }^{[16]}$ 报道了由于侧链的空间位阻作用较小, DBP 比 DEHP 降解 得更快, 因此生成 MBP 的速度也较 MEHP 快. 然而 MBP 和 MEHP 的降解速率是相当的. 由此可以推测沉积 物中 MBP 与 DBP 的浓度比值 (即 MBP/DBP) 要高于 MEHP 与 DEHP 的浓度比值 (即 MEHP/DEHP). 经计 算, 本研究中 MBP/DBP 确实大于 MEHP/DEHP.

除爱晚湖芦苇根中 MBP 和 MEHP 的浓度没有显著差异外, MEHP 在根中的浓度均明显高于 MBP, 表明 MEHP 比 MBP 有较强的根富集能力. 同一种植物单生时, 根中 PAMs 浓度均大于混生. 青年湖中, 香蒲根中 PAMs 浓度明显高于芦苇, 表明香蒲吸收 PAMs 的能力较强. 爱晚湖中, 香蒲根中 PAMs 浓度与芦苇根中浓度 的差异明显小于青年湖.

\section{5 结论}

几乎所有的样品中都能检测到 DBP 和 DEHP 以及它们的单酯代谢物, 并且 DBP 和 DEHP 的浓度比相 应的单酯代谢物高 1 2 个数量级. 沉积物和根样品中 DEHP 的浓度均高于 DBP. 芦苇和香蒲根对 DEHP 的 $R C F$ 值大于 DBP.

当芦苇和香蒲单生时 (即青年湖), 芦苇根际沉积物中 PAEs 浓度 $\leqslant$ 香蒲根际沉积物, 并且芦苇根的 $R C F$ 值也小于香蒲. 当芦苇和香蒲混生时 (即爱晚湖), 芦苇根际沉积物中 PAEs 浓度 <香蒲根际沉积物, 而芦苇 根的 $R C F$ 值 $\geqslant$ 香蒲根, 与青年湖中不同. 青年湖香蒲根际沉积物中 PAEs 的浓度小于爱晚湖, 而香蒲根的 $R C F$ 值与此刚好相反. 芦苇根对这两种 PAEs 的富集没有这种关系, 这可能是由于这两个湖中芦苇的生长状 
况不同,与香蒲混生时,芦苇的生长受到抑制.

对 PAEs 的单酯代谢产物分析发现,沉积物中 MBP/DBP 大于 MEHP/DEHP. MEHP 在根中的浓度多数情况高 于 MBP, 表明 MEHP 比 MBP 具有较强的根富集能力. 同种植物单生时,根中 PAMs 浓度均大于或等于混生.

上述结果表明,芦苇、香蒲这两种湿地植物单生或混生时根际 PAEs 及其单酯代谢物呈现出明显不同的 分布特征. 由于野外环境变因较多, 且无法对污染物的迁移转化进行定量, 因此后续还需加大现场采样力度 以及进行实验室受控研究,进一步探寻影响这类污染物根际分布的内在机制.

\section{6 参考文献}

[ 1 ] Matsumoto M, Hirata-Koizumi M, Ema M. Potential adverse effects of phthalic acid esters on human health: A review of recent studies on reproduction. Regulatory Toxicology and Pharmacology, 2008, 50(1) : 37-49.

[ 2 ] Staples CA, Peterson DR, Parkerton TF et al. The environmental fate of pathalate esters: A literature review. Chemosphere, 1997, 35(4) : 667-749.

[ 3 ] Liu M, Yang Y, Xu S et al. HCHs and DDTs in salt marsh plants (Scirpus) from the Yangtze estuary and nearby coastal areas, China. Chemosphere, 2006, 62(3): 440-448.

[ 4 ] Miglioranza K, De Moreno J, Moreno VJ. Organochlorine pesticides sequestered in the aquatic macrophyte Schoenoplectus californicus (C. A. Meyer) Soják from a shallow lake in Argentina. Water Research, 2004, 38(7) : 1765-1772.

[ 5 ] Toyama T, Sato Y, Inoue D et al. Biodegradation of bisphenol A and bisphenol F in the rhizosphere sediment of Phragmites australis. Journal of Bioscience and Bioengineering, 2009, 108(2) : 147-150.

[ 6 ] Cheema SA, Khan MI, Shen CF et al. Degradation of phenanthrene and pyrene in spiked soils by single and combined plants cultivation. Journal of Hazardous Materials, 2010, 177 (1/2/3) : 384-389.

[ 7 ] 李睿华,管运涛,何 苗等. 河岸混合植物带处理受污染河水中试研究. 环境科学,2006,27(4):651-654.

[ 8 ] Jonsson S, Boren H. Analysis of mono- and diesters of o-phthalic acid by solid-phase extractions with polystyrene-divinylbenzene-based polymers. Journal of Chromatography A, 2002, 963(1/2): 393-400.

[9] 国家环境保护总局《水和废水监测分析方法》编委会. 水和废水监测分析方法:第 4 版. 北京: 中国环境科学出版 社,2002:243-257.

[10] Barbour JP, Smith JA, Chiou CT. Sorption of aromatic organic pollutants to grasses from water. Environmental Science and Technology, 2005, 39(21) : 8369-8373.

[11] Chi J. Phthalate acid esters in Potamogeton crispus L. from Haihe River, China. Chemosphere, 2009, 77 (11) : 48-52.

[12]卢豪良,严重玲. 秋茄根系分泌物低分子量有机酸及其对重金属生物有效性的影响. 生态学报, 2007,27(10): 4173-4181.

[13] Cousins I, Mackay D. Correlating the physical-chemical properties of phthalate esters using the "three solubility" approach. Chemosphere, 2000, 41(9) : 1389-1399.

[14] Wong CS, Capel PD, Nowell LH. National-scale, field-based evaluation of the biota-sediment accumulation factor model. Environmental Science and Technology, 2001, 35(9) : 1709-1715.

[15] Srivastava A, Sharma VP, Tripathi R et al. Occurrence of phthalic acid esters in Gomti River sediment, India. Environmental Monitoring and Assessment, 2010, 169(1/2/3/4) : 397-406.

[16] Otton SV, Sura S, Blair J et al. Biodegradation of mono-alkyl phthalate esters in natural sediments. Chemosphere, 2008, 71(11) : 2011-2016. 